\title{
Multibody Simulation of an Electromagnetic Actuator for a Gentle Blood Pump Mechanism
}

\author{
Sebastian Pech, Heiko Rathmann, René Richter, Jens Lienig \\ Dresden University of Technology, Institute of Electromechanical and Electronic Design (IFTE) \\ Helmholtzstraße 18, 01062 Dresden, Germany \\ sebastian.pech@outlook.com; heiko-rathmann@web.de; rene.richter@tu-dresden.de; jens.lienig@tu-dresden.de
}

\begin{abstract}
In many blood-pump applications, the blood damage caused by mechanical stress limits the maximum operating time. In this paper, we introduce a novel pumping principle to reduce the mechanical stress within the pump. The principle is based on positive displacement, but, in contrast to common roller pumps, the flexible tube is stimulated by the eccentric motion of a shock head without complete tube occlusion. An MBS model (multibody simulation) is presented to simulate the stimulation of the flexible tube by the shock head. The model is based on the pump design and consists of several rigid, and elastic body elements. We conduct an FEM simulation of the electromagnetic actuator to compute a 3D map of the acting force vector that stimulates the MBS model. Finally, the generated eccentric motion is transmitted to the shock head via a bending rod. The shock-head trajectory can thus be simulated as a function of the electromagnetic actuator current. We validate the passive response of the MBS model by measuring the static displacement caused by a static force and the natural frequency of the experimental pump setup. In a second experiment the trajectory of the shock head is investigated to validate the complete MBS model stimulated by the 3D force-vector map.
\end{abstract}

Keywords: Blood Pump, Hose Pump, Electromagnetic Actuator, Multibody Simulation.

\section{Introduction}

Nowadays, modern medicine uses blood pumps in extracorporeal blood circulation systems, dialysis systems and organ perfusion systems. In all applications, the purpose of blood pumps is to transport blood as gently as possible to avoid damaging blood cells. The characteristic structure of an extracorporeal circulation system is shown in Fig. 1. This circuit is usually used for extracorporeal life support (ECLS), for example, extracorporeal membrane oxygenation (ECMO). One of the main system components is the oxygenator, where the gas exchange occurs. Roller pumps or centrifugal pumps are typically used to run the circulation and to perfuse the oxygenator with blood.

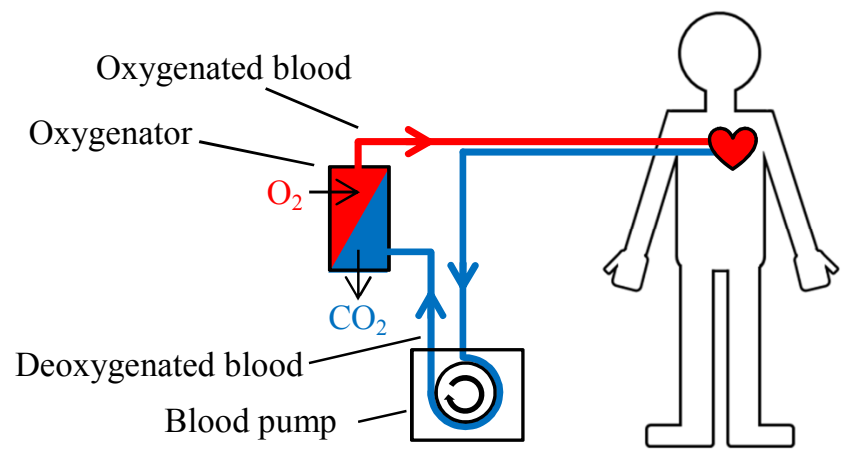

Fig. 1: Extracorporeal circulation according to [1].

During the extracorporeal circulation, the blood is subjected to mechanical stress and impacted by foreign surfaces [2], [3]. The most notable damage mechanism is called hemolysis. Due to the mechanical stress inside the blood pump, the red blood cells (erythrocytes) are damaged or destroyed. This blood damage limits the maximum application time for blood pumps. 
Roller pumps are typically deployed in life-support machines, for dialysis treatment and in organ perfusion systems. The operating time for these peristaltic pumps is limited to several hours. Rotating rollers inside the housing squeeze a flexible tube. Tube occlusion causes mechanical stress and hemolysis.

With a typical application time of several days, centrifugal pumps are commonly used, as well. They are classified as flow machines. Due to their operating principle, centrifugal pumps are not self-priming. Inside the housing a fast rotating impeller accelerates the blood. This causes a high velocity gradient which exposes the blood to high mechanical stress, and causes hemolysis [4].

To mitigate blood damage and increase the application time, a novel pumping principle is needed. By using a nonocclusive pumping principle the mechanical stress can be decreased [5]. To avoid additional blood damage the pump mechanism is designed without valves. Direct contact between fluid and moving parts should be avoided to minimize foreign-surface contact with the fluid. To ensure safe operation of organ perfusion systems, a maximum back pressure of $30 \mathrm{kPa}$ and a flow rate of $1.5 \mathrm{l} / \mathrm{min}$ are key design goals.

\section{Pumping Principle}

Fig 2. depicts a schematic of a roller pump. It consists of a flexible tube and a roller. The eccentric motion of the roller completely squeezes the flexible tube. This tube occlusion is necessary to generate fluid flow inside the flexible tube by means of positive displacement. Without complete tube occlusion, the reverse flow would decrease the regular flow because of the slow roller motion $(f<5 \mathrm{~Hz})$.

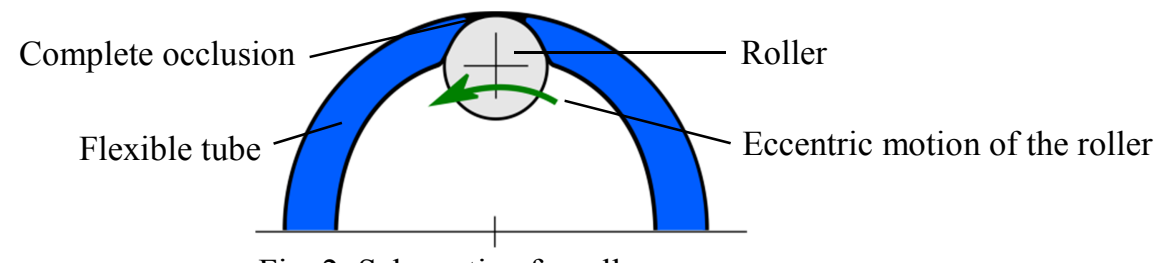

Fig. 2: Schematic of a roller pump.

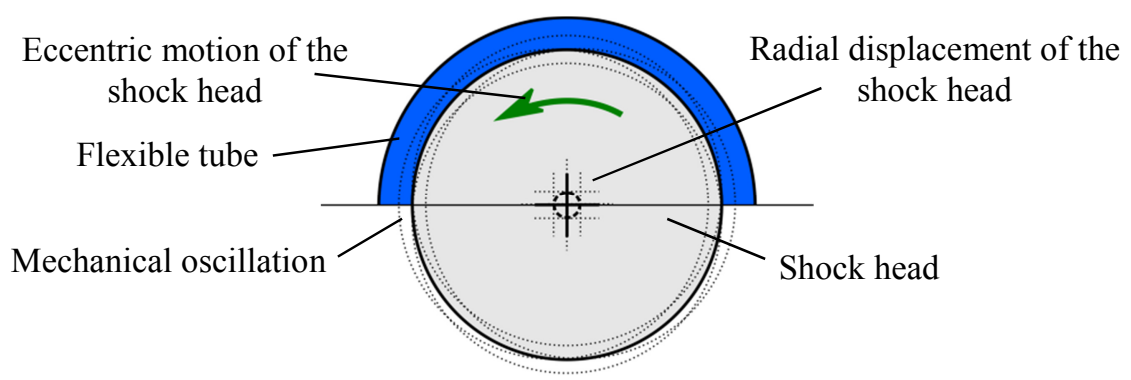

Fig. 3: Schematic of the non-occlusive pumping principle.

The new pumping principle is visualized in Fig. 3. Instead of the roller, a shock head stimulates the flexible tube. A positive displacement pumping principle is deployed here, too. In contrast to common roller pumps, the tube is not completely occluded while pumping. The flexible tube is stimulated by mechanical oscillations, generated by an electromagnetic actuator (not shown in Fig. 3). In this case, the tube is only slightly occluded and there is a throttled reverse flow due to the rapid eccentric motion of the shock head $(f \geq 50 \mathrm{~Hz})$. This meets the design goals of reducing the mechanical stress and hemolysis inside the pumped fluid.

\section{Actuator}

We have chosen a rotationally symmetric pump section to implement the pumping principle described above. The flexible tube can be arranged in windings around the shock head. The mechanical oscillations are generated by a circulating and oscillating force vector. This circulating force vector can easily be generated with an electromagnetic actuator. The longitudinal section of the pump is shown in Fig. 5. In the stacked design the pump section is located on top of the electromagnetic actuator. 


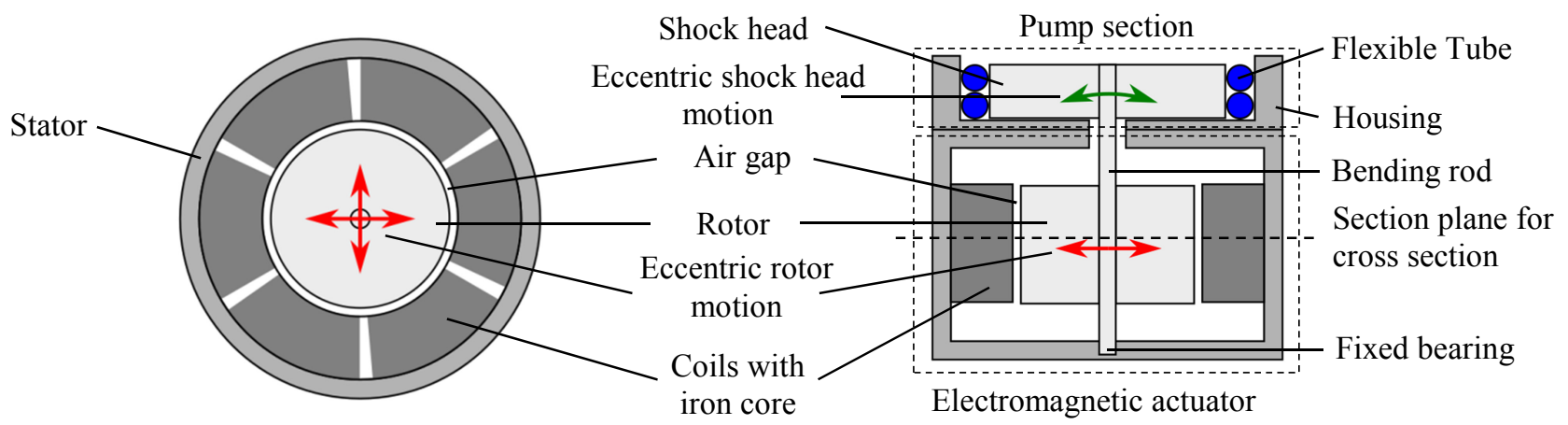

Fig. 4: Cross-section of the electromagnetic actuator according to [6], [7].

Fig. 5: Longitudinal section of the pump according to [8].

Fig. 4 shows the electromagnetic actuator cross-section. The main components are the stator and the rotor. Six rotationally symmetric coils with a closed iron core [6], [7] are arranged inside the stator. The rotor is positioned in the middle of the stator. By driving the coils with a phase-shifted coil current, the circulating force vector is generated by the air gap. This results in oscillations produced by the eccentric rotor motion with respect to the centre of the stator. In the next step, the eccentric rotor motion is transmitted through a bending rod to the shock head. The bending rod is attached to the actuator housing by a fixed bearing, thus preventing rotor rotation. The rotor eccentric motion bends the rod and transfers the oscillations to the shock head. The bending rod also acts as a spring, which, with its restoring force, centres the rotor. Finally, the shock head oscillates eccentrically with respect to the centre of the pump section, and the flexible tube is stimulated as described above.

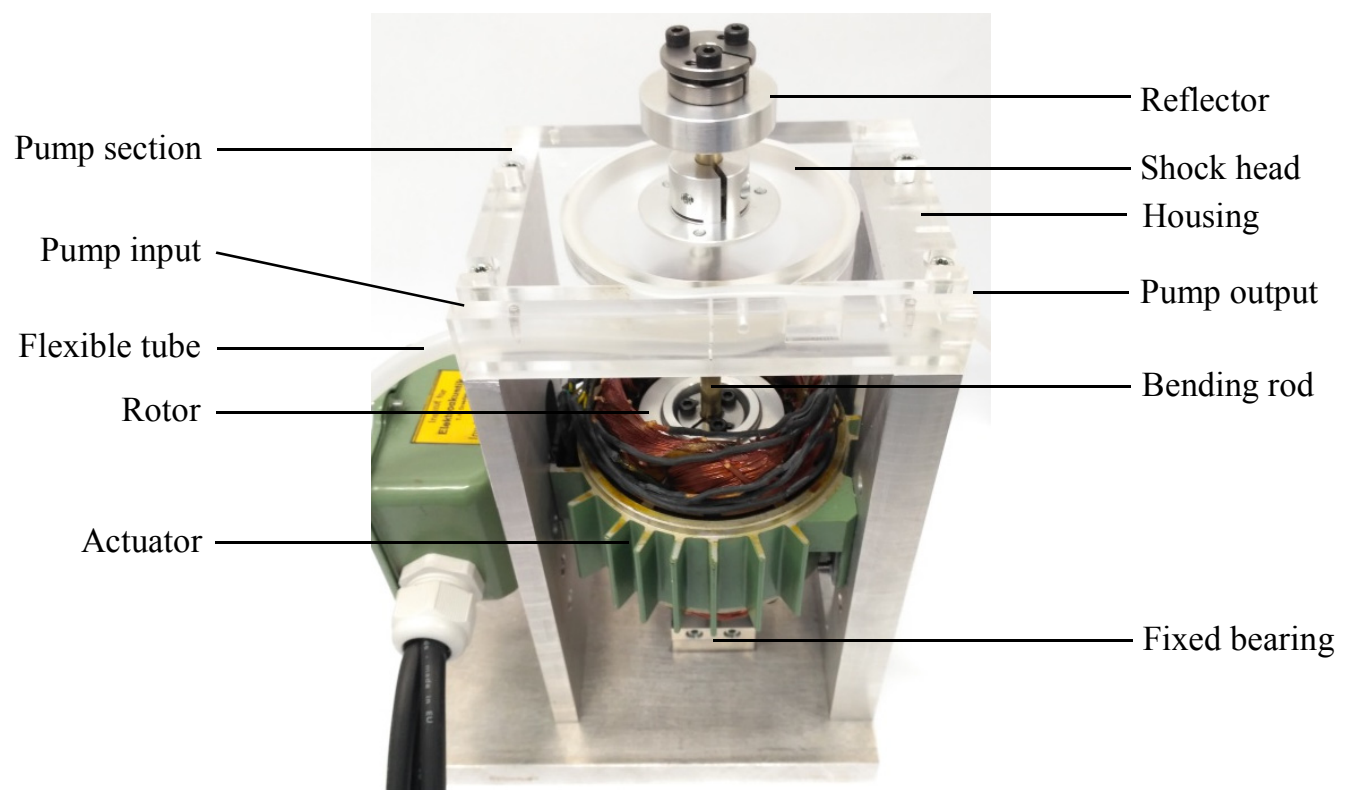

Fig. 6: Experimental pump setup.

In Fig. 6, the experimental pump setup with the dimensions $150 \mathrm{~mm}$ x $110 \mathrm{~mm}$ x $240 \mathrm{~mm}$ is illustrated. The bending rod (diameter $8 \mathrm{~mm}$ ) connects the electromagnetic actuator to the pump section. In the gap between the shock head and the pump section housing, the flexible silicone tube (5 mm inner diameter, $1 \mathrm{~mm}$ wall thickness) is arranged in two windings. Stimulated by the eccentric motion of the shock head, the fluid is pumped from the pump input on the left to the pump output on the right of the pump section. This experimental pump setup currently achieves a maximum flow rate of $0.25 \mathrm{l} / \mathrm{min}$ and a maximum backpressure of $20 \mathrm{kPa}$. 


\section{Simulation}

We simulate the motion of the moving parts (rotor, shock head and bending rod) as a function of the coil current in the electromagnetic actuator. The simulation structure is visualized in Fig. 7. The force vectors $F_{x}$ and $F_{y}$, which stimulate the rotor, are computed by an FEM simulation (finite element method) of the electromagnetic actuator.

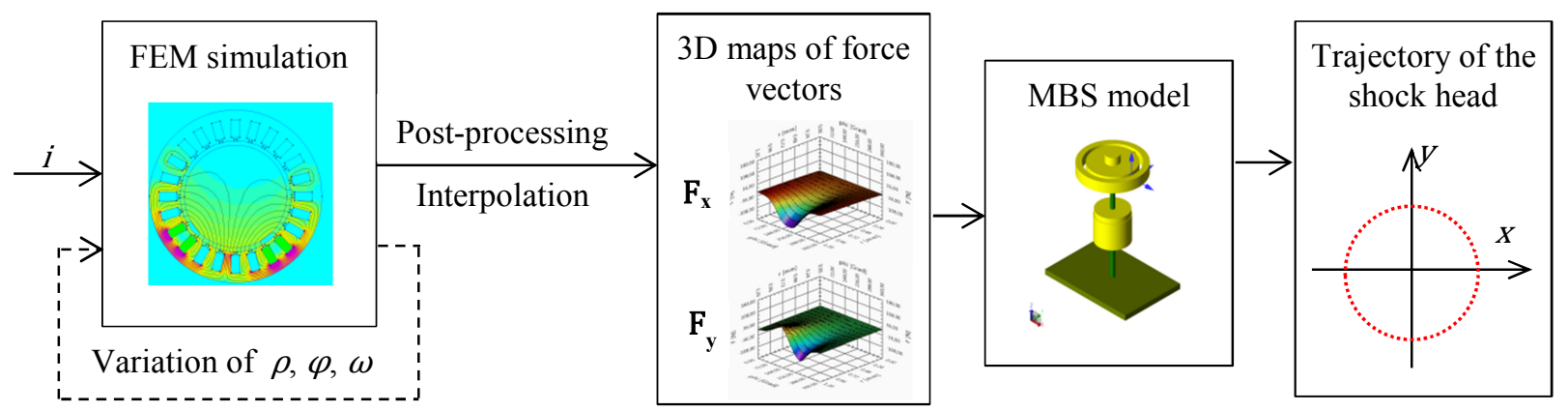

Fig. 7: Simulation structure.

Next, we imported these vectors into the MBS model (multibody simulation) to stimulate the rotor as described in Section 3. Finally, in the MBS model the bending rod transmits the motion to the shock head, and the shock-head trajectory can be simulated.

\subsection{FEM Simulation}

In the FEM simulation the rotor position is varied with respect to a cylindrical coordinate system. All rotor positions can thus be described with the radial displacement $\rho$ and the angle $\varphi$ (Fig. 8).

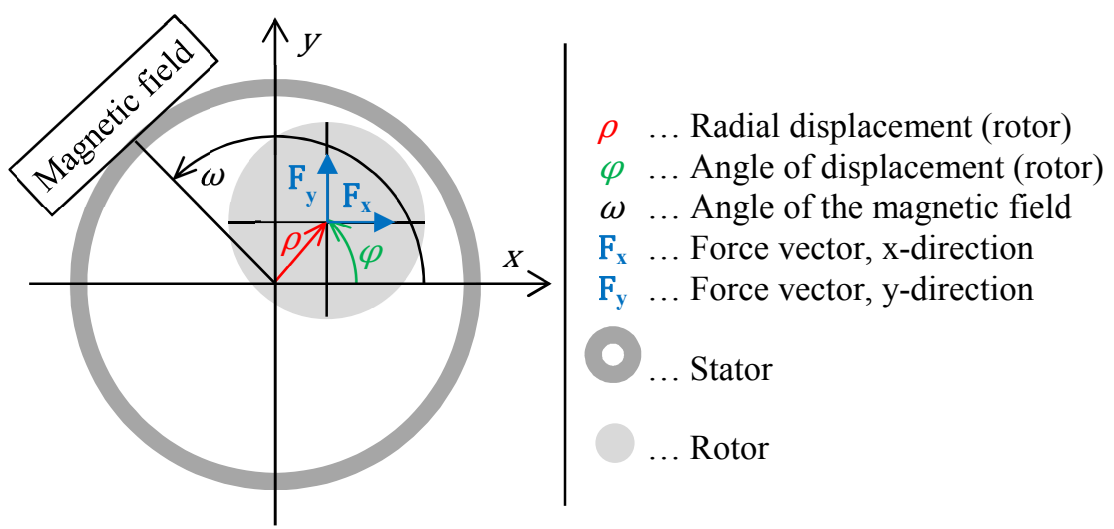

Fig. 8: FEM simulation parameters.

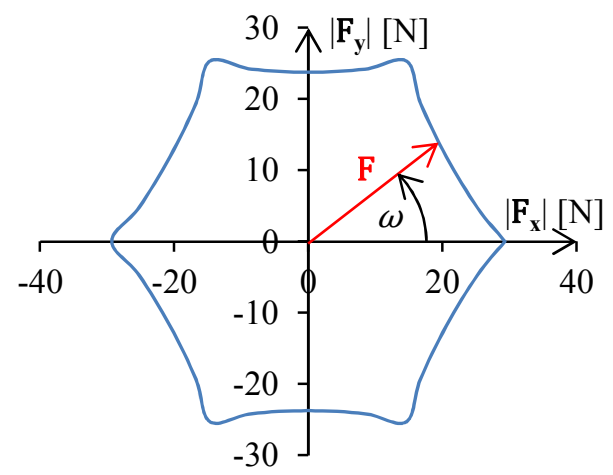

Fig. 9: Force vector $\mathrm{F}$ at $\hat{\imath}=1 \mathrm{~A}$ and $f=50 \mathrm{~Hz}$ for $\rho=0 \mathrm{~mm}$ and $\varphi=0^{\circ}$. 
The angle $\omega$ is varied, as well. It represents the direction of the magnetic field, which is generated by the coil current $i$. By varying $\rho, \varphi$ and $\omega$, the force vectors $\mathrm{F}_{\mathrm{x}}$ and $\mathrm{F}_{\mathrm{y}}$ can be calculated for every rotor position and every field angle $\omega$. Finally, two $3 \mathrm{D}$ maps of the force vectors $\mathrm{F}_{\mathbf{x}}$ and $\mathrm{F}_{\mathbf{y}}$ are generated in post-processing and interpolation steps. The resulting force is calculated as follows:

$$
\mathbf{F}=\mathbf{F}_{\mathbf{x}}(\rho, \varphi, \omega)+\mathbf{F}_{\mathbf{y}}(\rho, \varphi, \omega)
$$

As an example, the resulting force vector $F$ is shown in Fig. 9 as a combination of the force vectors $F_{\mathbf{x}}$ and $F_{\mathbf{y}}$. The chart represents $\mathrm{F}$ for a current amplitude of $\hat{\imath}=1 \mathrm{~A}$ and $f=50 \mathrm{~Hz}$ and a fixed rotor in the centre of the stator $(\rho=0 \mathrm{~mm}$ and $\varphi=0), \omega=(0 \ldots 2 \pi)$.

\subsection{Multibody Simulation}

The MBS model was simulated with the SimulationX software package (version 3.8, ESI ITI GmbH). In Fig. 10 the pump design and the structure of the MBS model are pictured. The MBS model is based on the actuator design. The main components are rigid body elements (housing, rotor, shock head and reflector) and elastic body elements, representing the sections of the bending rod.

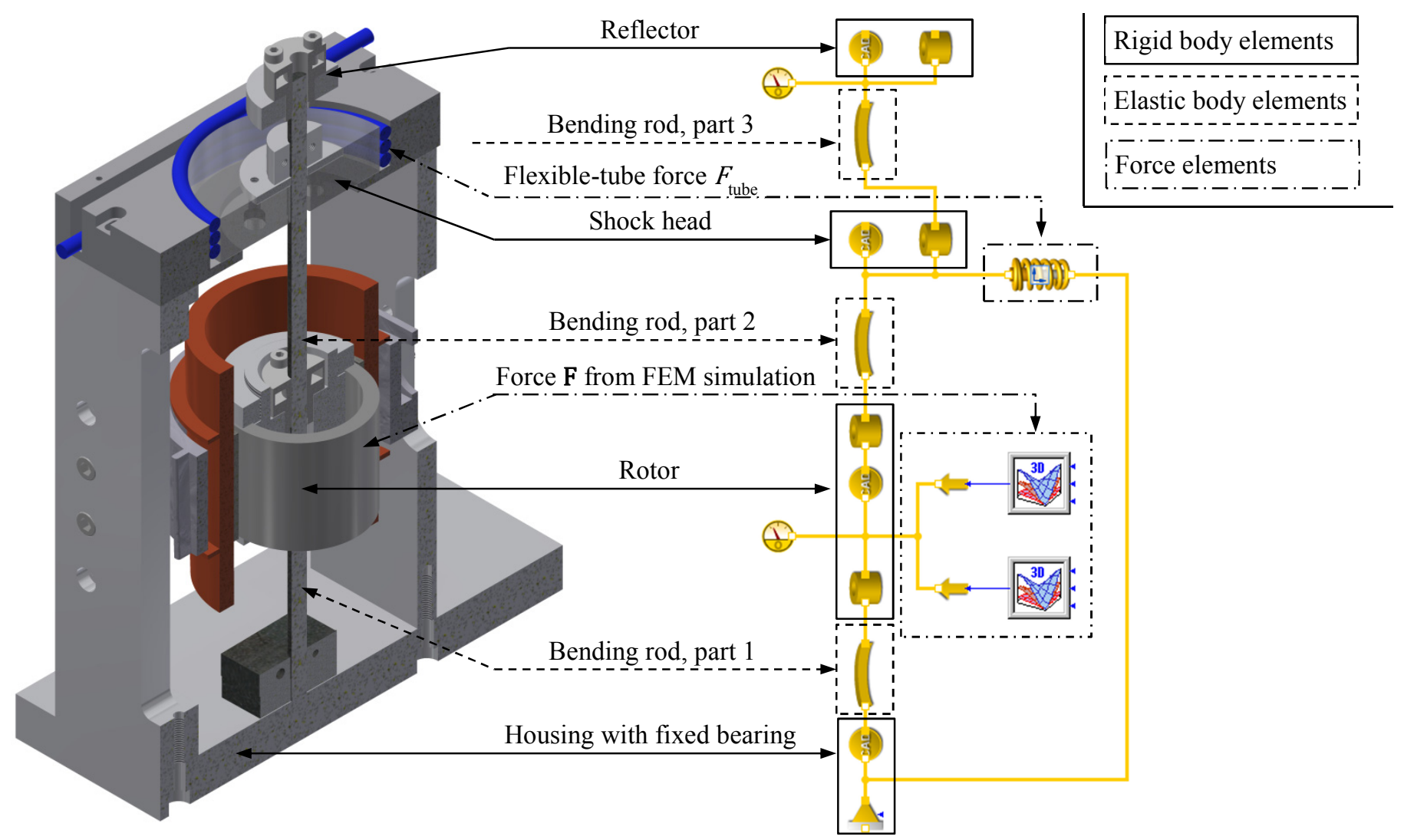

Fig. 10: Partial section of the 3D pump design model with associated MBS-model structure.

There are also force elements which represent the stimulating force $\mathbf{F}$ from the FEM simulation. These elements are connected to the rotor and coupled to the rotor kinematic sensor. The rotor position is thus used as input for the 3D maps of the force vector $\mathbf{F}$. The pump's mechanical load is generated by the force of the flexible tube $F_{\text {tube. }}$ It is defined as the force required to squeeze the flexible tube by a set amount. The force $F_{\text {tube }}$ is connected to the shock head. A reflector is fitted to the upper part of the bending rod. Shock-head motion can be measured with this part using laser distance sensors. A kinematic sensor is coupled with the reflector in the MBS model. This allows us to compute and compare the reflector motion with the reflector and shock-head motions in the experimental pump setup. 


\section{Results}

We carried out a number of experiments to verify the function of the MBS model. First, the passive behaviour of the moving parts are investigated. Second, the MBS model is stimulated by the force from the FEM simulation to investigate the complete system response. The force model for the flexible tube is currently being developed; therefore, in all experiments $F_{\text {tube }}=0$.

\subsection{Static Force and Natural Frequency}

The static and dynamic behaviour of the bending rod and the coupled elements (rotor, shock head and reflector) are investigated in this section. In these experiments the electromagnetic actuator is deactivated $(F=0)$ and the reflector displacement is measured with a laser distance sensor (Keyence, LK-G82, LK-GD500). In the first experiment, two different static forces are coupled successively to the bending rod. Thus, the shock head and the reflector are displaced. The experimental setup is shown in Fig. 11. In Table 1 the measured reflector displacement is compared with the computed displacement from the MBS model. The mean $\bar{X}_{\text {meas }}$ and the standard deviation $\sigma_{\text {meas }}$ are calculated based on 10 single displacement measurements.

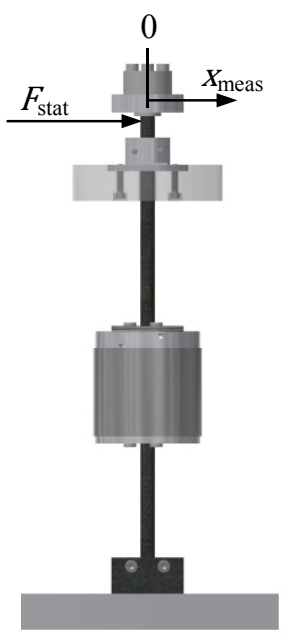

Fig. 11: Experimental setup: static displacement $\left(F_{\text {stat }} \neq 0\right)$ and natural frequency $\left(F_{\text {stat }}=0\right)$.

Table 1: Displacement with static force.

\begin{tabular}{|c|c|c|c|c|c|}
\hline$F_{\text {stat }}$ & $X_{\text {MBS }}$ & $\bar{X}_{\text {meas }}$ & $\sigma_{\text {meas }}$ & \multicolumn{2}{|c|}{ Deviation related to measurement } \\
\hline $4.96 \mathrm{~N}$ & $0.832 \mathrm{~mm}$ & $0.943 \mathrm{~mm}$ & $0.011 \mathrm{~mm}$ & $-0.111 \mathrm{~mm}$ & $-11.77 \%$ \\
\hline $9.83 \mathrm{~N}$ & $1.648 \mathrm{~mm}$ & $1.834 \mathrm{~mm}$ & $0.019 \mathrm{~mm}$ & $-0.186 \mathrm{~mm}$ & $-10.14 \%$ \\
\hline
\end{tabular}

Table 2: Natural frequency.

\begin{tabular}{|c|c|c|c|c|}
\hline$f_{\text {nat_MBS }}$ & $\bar{f}_{\text {nat_meas }}$ & $\sigma_{\text {nat_meas }}$ & \multicolumn{2}{|c|}{$\begin{array}{c}\text { Deviation related } \\
\text { to measurement }\end{array}$} \\
\hline $23.50 \mathrm{~Hz}$ & $21.61 \mathrm{~Hz}$ & $0.24 \mathrm{~Hz}$ & $1.89 \mathrm{~Hz}$ & $8.75 \%$ \\
\hline
\end{tabular}

The static displacement experiment proved that the deviation between the MBS model and the measured values is less than $10.77 \%$. The standard deviation of the measurements is about $0.019 \mathrm{~mm}$. Thus, without the impact of the flexible tube and the electromagnetic actuator, the static behaviour of the MBS model is validated.

In the second experiment, the system's natural frequency is measured via the laser distance sensor. The experimental setup is the same as the setup shown in Fig. $11\left(F_{\text {stat }}=0\right)$. After initial mechanical stimulation of the bending rod, the system oscillates at its natural frequency. Based on the laser distance signal, the natural frequency can be calculated. The results of 10 single measurements are shown in Table 2 . The natural frequency can be computed in the MBS model with a 
deviation of $8.75 \%$ from the measured frequency. In this experiment the natural frequency is measured with a standard deviation of $0.24 \mathrm{~Hz}$. Both experiments show that the bending rod reacts slightly stiffer in the MBS model than in the experiment $\left(f_{\text {nat_MBS }}>\bar{f}_{\text {nat_meas }}\right.$ and $\left.x_{\mathrm{MBS}}<\bar{X}_{\text {meas }}\right)$.

\subsection{Electrical Stimulation}

In this experiment, the MBS model is stimulated with the 3D maps of force vectors from the FEM simulation $(\hat{\imath}=1 \mathrm{~A}$ and $f=50 \mathrm{~Hz}$ ). In Fig. 12 the simulated trajectory of the centre of the reflector is plotted in blue. To verify this trajectory, two orthogonally arranged laser distance sensors (Keyence, LK-G82, LK-G32, LK-GD500) are used to measure the reflector displacement in the $x$ and $y$ directions. With a combination of both sensors, the position of the reflector centre $\left(x_{\mathrm{c}}\right.$ and $y_{\mathrm{c}}$ ) can be calculated [1]. In Fig. 12 the experimentally measured reflector trajectory is plotted in red.

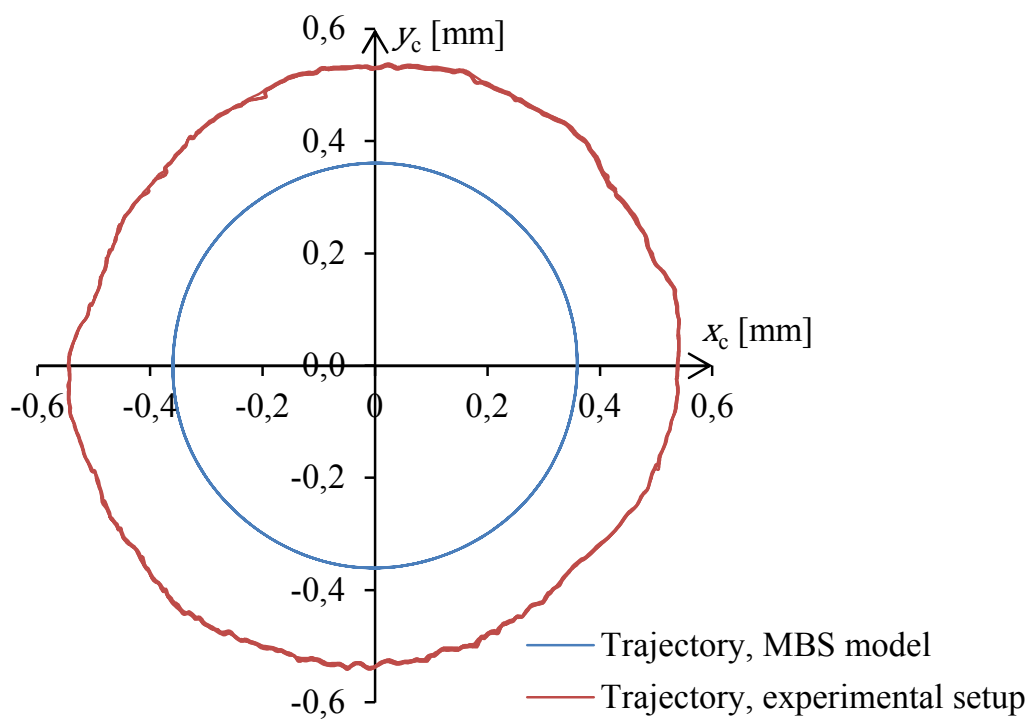

Fig. 12: Reflector trajectory, MBS model vs. experiment, sampling period: $0.1 \mathrm{~s}$.

The radial reflector displacement of the MBS model is $0.36 \mathrm{~mm}$. The measured trajectory of the experimental setup shows a radial displacement of $0.53 \mathrm{~mm}$. A comparison of the trajectories reveals that the radial reflector displacement varies on average by approximately $0.17 \mathrm{~mm}$ between the MBS model and the experiment. This corresponds to a simulated reflector oscillation amplitude that is around $32.1 \%$ smaller than that measured in the experiment. This deviation must be remedied in order to validate the MBS model. We suspect that the discrepancy is due to the bending behaviour of the bending rod in the MBS model and the bearing of the bending rod in the experimental setup. Compared with the MBS model, the rod is not ideal fixed in the experimental setup. Both result in a lager radial displacement of the experimental setup compared to the MBS model. This corresponds with the stiffer bending rod reaction of the MBS model as a result of the passive behaviour experiments.

\section{Discussion}

We developed an MBS model to simulate the dynamics of the moving parts in the blood pump. The results of the displacement with static force show that the passive static response of the MBS model agrees with the experimental setup. The natural frequency of the MBS model also corresponds with the experimental setup. The bending behaviour of the rod needs to be revised to obtain a comparable shock-head trajectory. In addition, the bearing of the bending rod must be optimized to ensure a completely fixed bearing, which is comparable with the bearing in the MBS model. Finally, the force model of the flexible tube must be developed to complete the MBS model. 


\section{Conclusion and Outlook}

In this paper a new pumping principle, based on positive displacement, is introduced. To decrease the mechanical stress and to avoid hemolysis, the pump works without completely occluding the flexible tube. We developed an MBS model, based on the actuator design, to simulate the mechanical stimulation of the flexible tube. An FEM simulation of the electromagnetic actuator is used to compute a force vector, which is coupled with the MBS model via 3D maps. A complete mechanical model of the pump is thus available. As shown experimentally, the passive behaviour of the MBS model is validated by measuring the static displacement at static force, and by measuring the natural frequency. The bending behaviour of the rod and its bearing in the experimental setup must be revised to validate the electrical stimulation of the MBS model.

After validating the MBS model, the shock-head trajectory can be used to stimulate a hydraulic model. Within this model the pumping principle itself is to be modelled. By coupling the FEM simulation, the MBS model and the hydraulic model, the pump characteristics can be computed based on the electromagnetic-actuator current.

A complete pump model will then be available which can be used to optimize the pump configuration to reduce blood damage and to increase the maximum application time of blood pump operations.

\section{Acknowledgements}

This work is supported by the European Social Fund (ESF) and the Free State of Saxony, Germany.

\section{References}

[1] S. Pech, et al, "Electromagnetic Actuator of a Gentle Pump Mechanism for Blood Transport," in Proceedings of the $59^{\text {th }}$ Ilmenau Scientific Colloquium (59 $9^{\text {th }}$ IWK), Ilmenau, Germany, 2017. ISBN:978-3-86360-160-7, urn:nbn:de:gbv:ilm1-2017iwk-020:2.

[2] D. Arora, M. Behr, M. Pasquali, "Blood damage measures for ventricular assist device modelling," in Moving Boundaries VII-Computational Modelling of Free and Moving Boundary Problems, A. A. Mammoli, C. A. Brebbia, ed. Southampton: WIT Press, 2003, pp. 129-138.

[3] H.-D. Polaschegg, "Red Blood Cell Damage from Extracorporeal Circulation in Hemodialysis," Seminars in Dialysis, vol. 22, no. 5, pp. 524-531, 2009.

[4] K. H. Fraser, et al, "A Quantitative Comparison of Mechanical Blood Damage Parameters in Rotary Ventricular Assist Devices: Shear Stress, Exposure Time and Hemolysis Index," Journal of Biomechanical Engineering, vol. 134, no. 8, pp. 081002-1-081002-11, 2012.

[5] Y. Tamari, et al, "A Dynamic Method for Setting Roller Pumps Nonocclusively Reduces Hemolysis and Predicts Retrograde Flow," ASAIO J., vol. 43, no 1, pp. 39-52, 1997.

[6] A. R. Druschke, et al, "Elektromechanische Wandler nach dem Wanderfeldprinzip und ihre Anwendungsmöglichkeiten insbesondere in der Medizintechnik," messen-steuern-regeln, Verl. Technik, vol. 23, no. 8, pp. 432435,1980

[7] A. R. Druschke, M. Peschel, "Elektrisch betriebene Pumpe," German Patent DD156386C2, February 13, 1981

[8] S. Pech et al, "Elektrisch betreibbare Schlauchpumpe," German Patent Reference No. 102017114 950.3, Registered at DPMA July 5, 2017. 